\title{
Output Signal Power Analysis in Erbium-Doped Fiber Amplifier with Pump Power and Length Variation Using Various Pumping Techniques
}

\author{
S. Semmalar' ${ }^{1}$ and S. Malarkkan ${ }^{2}$ \\ ${ }^{1}$ SCSVMV University, Kanchipuram, India \\ ${ }^{2}$ Manakula Vinayagar Institute of Technology, Puducherry 605 107, India \\ Correspondence should be addressed to S. Semmalar; subbusem@gmail.com
}

Received 5 June 2013; Accepted 27 June 2013

Academic Editors: H. J. De Los Santos and H. L. Hartnagel

Copyright (c) 2013 S. Semmalar and S. Malarkkan. This is an open access article distributed under the Creative Commons Attribution License, which permits unrestricted use, distribution, and reproduction in any medium, provided the original work is properly cited.

\begin{abstract}
The scope of this paper is to analyze the output signal power with pump power and length variation in cascaded EDFA simulation model performance. This paper describes the simulation model of Erbium-Doped Fiber Amplifier (EDFA) of variable lengths (10 m, $50 \mathrm{~m}$, and $120 \mathrm{~m}$ ) with dual pumping techniques (dual forward pumping with two $980 \mathrm{~nm}$ wavelengths, dual forward and backward pumping with two $980 \mathrm{~nm}$ wavelengths) and Tri-pumping techniques. The simulation models consist of input source and pump power coupled by WDM coupler which gives optimized signal power in the above-mentioned simulation model. The simulation model consists of source with multiple wavelengths $(1520 \mathrm{~nm}-1618 \mathrm{~nm})$, pumping source with the wavelength $980 \mathrm{~nm}$, isolator, and filter. The resulting models accurately represent EDFA optimized output signal power. Simulation results show that choosing careful fiber length $120 \mathrm{~m}$ and pump power $1 \mathrm{~W}$ in dual pumping provided $0.07 \mathrm{~W}$ optimized output signal power compared to other pumping techniques.
\end{abstract}

\section{Introduction}

As the demand of high data speed networks is increasing, an answer to long distance communication system is optical communication systems which employ optical fiber that can be used as a medium for telecommunication and networking. The light propagates through the optical fiber with little attenuation compared to electrical cables. An optical amplifier is a device that amplifies an optical signal directly without the need to first convert it to an electrical signal in optical fiber communications. EDFAs are mostly used as preamplifiers with multichannel amplification without crosstalk and also multigigabit transmission rates by low bit errors [1].

Most important element of EDFA technology is the Erbium-Doped Fiber (EDF), which is a conventional silica fiber doped with Erbium. Erbium-doped fiber amplifiers have attracted the most attention because they operate in the wavelength region near $1.55 \mu \mathrm{m}$. The deployment of EDFA in WDM systems has revolutionized the field of optical fiber communications and led to light wave systems with capacities exceeding $1 \mathrm{~Tb} / \mathrm{s}$.

1.1. Basic Principle of EDFA. Amplification in an Erbiumdoped fiber amplifier occurs through the mechanism of stimulated emission. When the Erbium is illuminated with light energy at a suitable wavelength (either $980 \mathrm{~nm}$ or $1480 \mathrm{~nm}$ ), it is excited to a long lifetime intermediate state level 2 following which it decays back to the ground state by emitting light within the $1500-1600 \mathrm{~nm}$ bands [2]

If light energy already exists within the $1500-1600 \mathrm{~nm}$ band, for example, due to a signal channel passing through the EDF, then this stimulates the decay process (so-called stimulated emission), resulting in additional light energy. A pumping signals can copropagate with an information signal or it can counter propagate. Thus, if a pump wavelength and a signal wavelength are simultaneously propagating through an EDF, energy transfer will occur via the Erbium from 
the pump wavelength to the signal wavelength, resulting in signal amplification. A wavelength far from the emission peak around $1530 \mathrm{~nm}$ has to improve the amplification characteristics of the L-band and C-band EDFA. An important issue is the selection of a proper pump wavelength or a suitable pumping configuration. The pump wavelength dependence of the amplification characteristics of the EDFA has been reported mainly in 800-, 980-, and 1480-nm bands, and now the 980- and 1480-nm bands are mostly used for the L-band and C-band EDFA's.

1.2. Signal Power in an EDFA. The output signal power is calculated as

$$
P_{\text {out }}=P_{\text {in }} \times G,
$$

where $G$ is the EDFA (amplifier) power gain and $P_{\text {in }}$ is the input signal power. The most important feature of the EDFA is gain as it determines the amplification of individual channels when a WDM signal is amplified [3]. The amplified output signal power is measured from the output line and is taken after the filter in the block diagram of Figure 1. And the input signal power is fixed as $0.001 \mathrm{mw}$. This amplified output signal power is degraded due to the Amplified Stimulated Emission (ASE) noise, and the output signal power increases due to the stimulated emission, and this is due to population inversion and population inversion is due to pumping power.

The gain of the EDFA is limited by the fact that there are a limited number of Erbium ions in the core. Increasing the pump power beyond the point where all the ions are excited cannot produce more gain, and thus saturation occurs. An erbium-doped amplifier can amplify light wavelength ranging from app $1500 \mathrm{~nm}$ to more than $1600 \mathrm{~nm}$.

Two such bands are in use today. One is the C-band (conventional band) which occupies the spectrum from $1530 \mathrm{~nm}$ to $1560 \mathrm{~nm}$ and the second is L-band (long wavelength band) which occupies the spectrum ranging from $1560 \mathrm{~nm}$ to $1610 \mathrm{~nm}$. Most EDFA work in the C-band. Noise is the second most important characteristic of an optical amplifier.

An erbium-doped fiber is the active medium of an EDFA. The two most important elements in order to produce high amplified output signal power in L-band EDFA are the length of EDF and the pump power both of which have been analyzed in this paper.

This paper is organized into six sections. Section 2 is a literature review of this work, while Section 3 presents the methodology and the proposed work. Section 4 demonstrates the model simulation details. Section 5 presents the results and discussions. Finally, the paper is concluded in Section 6.

\section{Literature Review}

This paper [1] presents a composite EDFA configuration which incorporates an optical isolator and investigated highly efficient amplifier configurations with high total gain and narrow ASE spectrum. This paper [4] designed the broadband EDFA using dual forward pumping and results to increased gain and gain bandwidth. This paper [5] proposed an EDFA pumped in the $660 \mathrm{~nm}$ and $820 \mathrm{~nm}$ bands wavelength gives

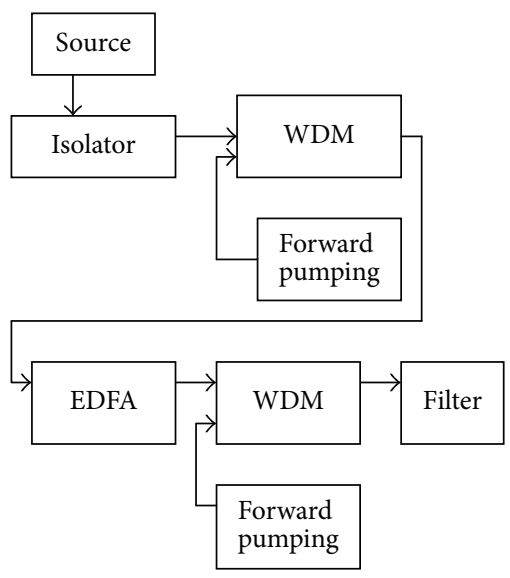

FIGURE 1: Block diagram of dual stage (forward) pumping.

enhanced gain. This paper [6] deals with amplifier's gain and noise power Which appear in the signal to-noise ratio expression are computed in terms of the internal parameters from simulations and are shown to contribute to amplifiers improvement. The paper [7] developed an analytic model for gain modulation in EDFAs. The analytic model was then used to explore the effect of mean input signal power (EDFA gain saturation) and dependence on signal wavelength. It was found that pump to signal modulation index increases with signal power (saturation), rising to a maximum and then decreasing as EDFAs become deeply saturated. The reverse is true of the signal to signal modulation index. The paper [8] proposed an average power analysis technique similar to that used for semiconductor optical amplifiers. In this paper [9] analyzed gain versus pump power for EDFA. This paper [10] allows network designers to determine the tolerances by which the signal power levels may deviate from their predesigned average values.

\section{Methodology}

In this work, the analysis of amplified signal power output from the simulation models of EDFA with dual forward pumping using wavelength $980 \mathrm{~nm}$ and dual forward and backward pumping using wavelength $980 \mathrm{~nm}$ with the pump power variation of $0.22 \mathrm{~W}, 0.62 \mathrm{~W}$, and $1 \mathrm{~W}$ with different EDFA lengths $(10 \mathrm{~m}, 50 \mathrm{~m}$, and $120 \mathrm{~m}$ ) has been simulated with WDM blocks, and a high performance approach is presented that has not been used in this manner before for such design. Erbium-doped fiber amplifiers (EDFAs) employed in Wavelength Division Multiplexing (WDM) systems have been shown to incur system impairment due to optimized signal output power $0.07 \mathrm{w}$ in dual pumping, $980 \mathrm{~nm}$ wavelengths compared to Tri-forward pumping techniques.

3.1. Applied Methodology. The applied methodology is based on dual forward pumping and dual forward and backward pumping and Tri-pumping approach. Each block in the architecture was added in the model and tested, and later those blocks were assembled and were added to compose 


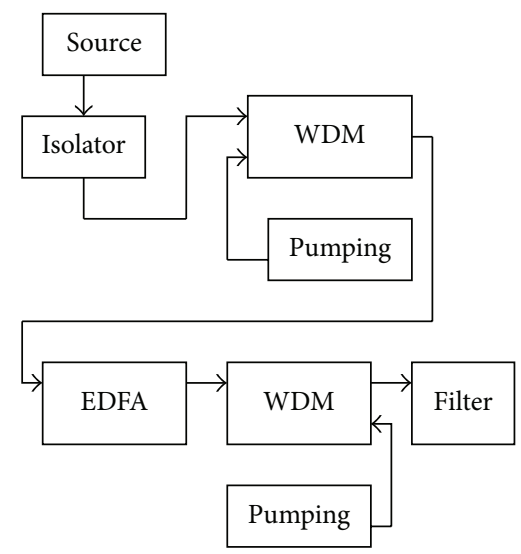

FIGURE 2: Block diagram of dual stage (forward-backward) pumping.

the complete system and then simulated and tabulated the parameter (output amplified signal power) values.

3.2. Proposed Work. Figure 1 shows block diagram of EDFA with dual forward pumping using wavelength $980 \mathrm{~nm}$, and Figure 2 shows block diagram of EDFA with dual forward and backward pumping using the wavelength of $980 \mathrm{~nm}$. The simulation model consists of the input source with different channels (1520 nm-1618 nm) whose output is given to the isolator.

Further the output of the isolator and the first pumping source (may be forward or backward) has been multiplexed using WDM technique. This multiplexed signal is given to the EDFA where the signal is amplified to improve the gain. A wavelength-division-multiplexing (WDM) technique combined with erbium-doped fiber amplifier (EDFA) is essential for realizing high capacity light wave transmission and flexible optical networks.

Recently, lots of problems in bidirectional EDFAs were investigated, and various structure schemes of the EDFA were reported to overcome the problems, such as back reflections [11]. Anautomatic gain control (AGC) function for bidirectional EDFAs, however, has been rarely reported.

This method has the advantage of providing optical fiber with few Erbium clusters because the Erbium is uniformly doped into silica soot preform in a vapor phase atmosphere. In order to attain highly efficient EDFAs, the three key factors outlined below must be considered.

The first is the Erbium concentration effect on Erbium cluster generation in silica-based glass [4]. Compared with unidirectional transmission, bidirectional transmission over a single fiber has the advantage of reducing not only the number of fiber link but also the number of passive components such as splitters and WDM multiplexers. It has already been confirmed that an increase in Erbium concentration causes deterioration in amplification efficiency [5].

In the previous block diagrams the Erbium inversion level increases whenever EDFA uses less distance with the same pump power. For example the pump signal power $1 \mathrm{w}$, the fiber inversion level increases with the EDFA length position

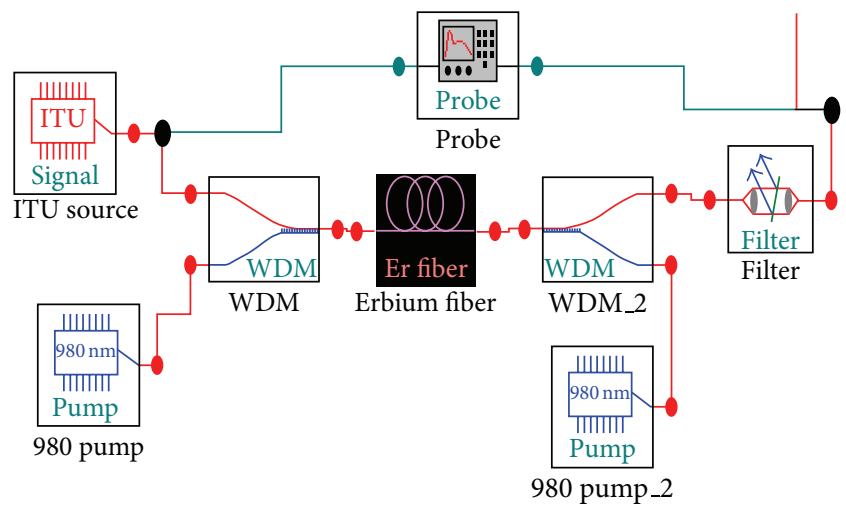

Figure 3: Simulated model of EDFA with dual forward and backward pumping scheme.

between $50 \mathrm{~m}$ to $120 \mathrm{~m}$, this shows if the fiber inversion level increases the output amplified signal also increases, that is an inversion level is depends upon the pump power and wavelength.

Isolator allows the forward propagation for that there is no reflected signal. Compared with single stage techniques the model has an advancement of not cascading pumping sources in unidirectional way and hence a reduction in compactness. Filter here is to remove the unwanted wavelengths.

\section{Model Simulation}

The simulation models, EDFA with dual forward and backward pumping using $980 \mathrm{~nm}$ twice and Tri-forward pumping with wavelengths $980 \mathrm{~nm}$, are shown in Figures 3 and 4 .

The parameters output amplified signal power has been measured with different pump powers $0.22 \mathrm{~W}, 0.62 \mathrm{~W}$, and $1 \mathrm{~W}$ and with different lengths $10 \mathrm{~m}, 50 \mathrm{~m}$, and $120 \mathrm{~m}$ from the simulation model and that has been tabulated and analyzed.

The gain spectrum of EDFAs can vary from amplifier to amplifier even when core composition is the same because it also depends on the fiber length. The main difference between forward and backward pumping technique is that in the later one pump power and the signal beam propagate in opposite directions as compared to the forward pumping scheme.

Wavelength-division multiplexing (WDM) technology employing erbium-doped fiber amplifiers (EDFAs) provides a platform for significant improvement in network bandwidth capacity, and WDM will play a dominant role in backbone infrastructure supporting the next generation high-speed networks. But due to complexity and variation in the output power level of various channels, a limitation regarding change in gain profile of each channel makes the system in appropriate for dense multichannels.

\section{Results and Discussions}

The simulation result shows output amplified signal power in watts when the input signal power is $0.001 \mathrm{mw}$. Simulation results indicate that the amplified signal power from the transmitter output increases when pump power increases, 


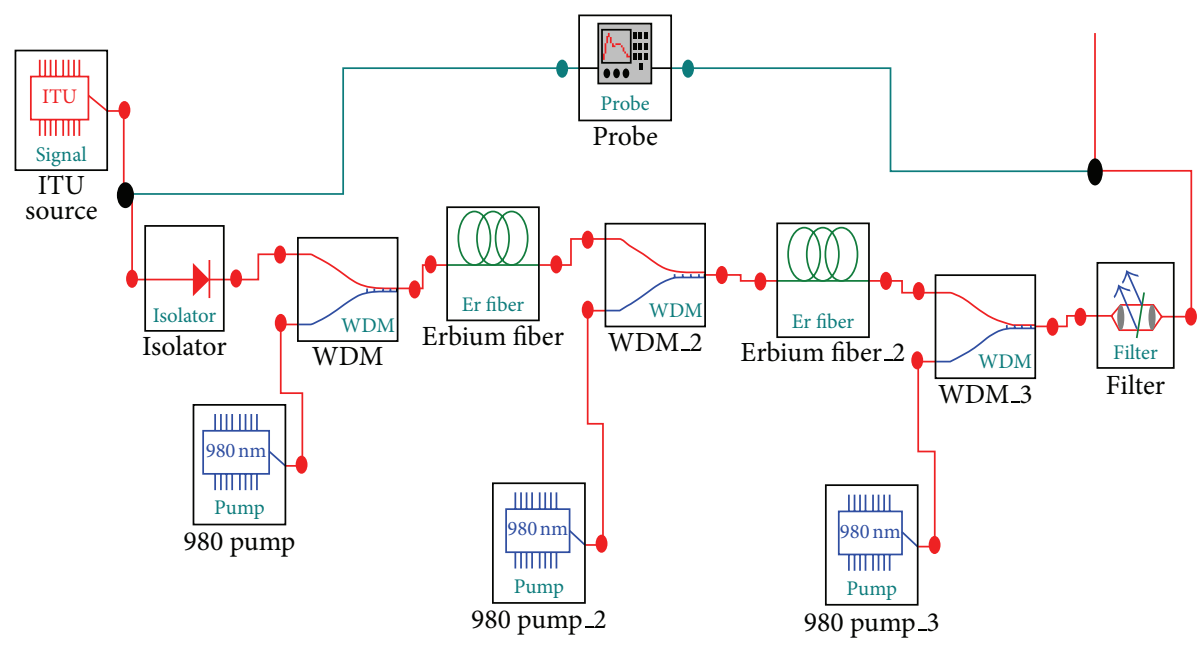

FIGURE 4: Simulation model of EDFA with Tri-forward pumping using $980 \mathrm{~nm}$ wavelength.

TABLE 1: Results: output amplified signal power of dual pumping with wavelength $980 \mathrm{~nm}$ for different pumping power and different EDFA length.

\begin{tabular}{|c|c|c|c|c|}
\hline \multirow[t]{2}{*}{ Pump power } & \multicolumn{3}{|c|}{$\begin{array}{c}\text { Output amplified signal power in dual pumping with wavelength } 980 \mathrm{~nm}(\mathrm{~W}) \\
\text { with the constant input signal power }=0.001 \mathrm{mw}\end{array}$} & \multirow[t]{2}{*}{ Remarks } \\
\hline & EDFA length $10 \mathrm{~m}$ & EDFA length $50 \mathrm{~m}$ & EDFA length $120 \mathrm{~m}$ & \\
\hline Pump power $=0.22 \mathrm{w}$ & 0.016 & 0.016 & 0.0155 & S/g power decreases \\
\hline Pump power $=0.62 \mathrm{w}$ & 0.038 & 0.042 & 0.045 & S/g power increases \\
\hline Pump power $=1 \mathrm{w}$ & 0.05 & 0.065 & 0.07 & S/g power increases \\
\hline Remarks & S/g power increases & S/g power increases & S/g power increases & \\
\hline
\end{tabular}

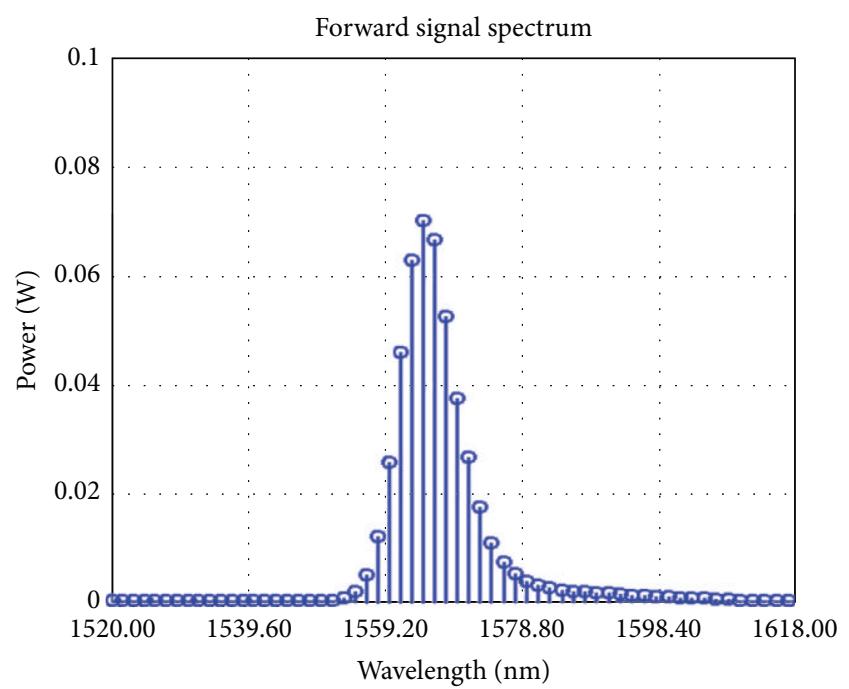

Figure 5: Amplified output Signal power with $980 \mathrm{~nm}$ pump and $120 \mathrm{~m}$ length.

but amplified signal power decreases when EDFA length increases in dual pumping technique with $980 \mathrm{~nm}$ as shown in Table 1.

Tables 1, 2, and 3 show that the simulated values are tabulated, amplified output signal power compared with

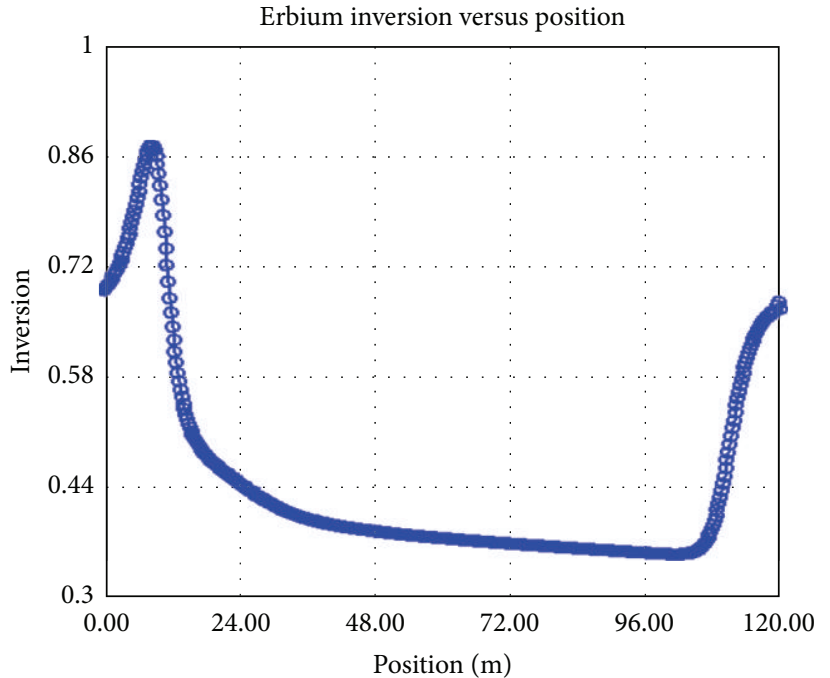

FIGURE 6: Erbium inversion with position in EDFA.

various pumping techniques for different pump power and EDFA length $=10 \mathrm{~m}, 50 \mathrm{~m}$, and $120 \mathrm{~m}$ with dual forward and dual forward and backward and Tri-forward pumping techniques (Figure 5).

Figure 6 shows the Erbium inversion level with position of EDFA distance in meters. The stimulated emission is due 
TABLE 2: Results: output amplified signal power of dual forward pumping with wavelength $980 \mathrm{~nm}$ for different pumping power and different EDFA length.

\begin{tabular}{|c|c|c|c|c|}
\hline \multirow[t]{2}{*}{ Pump power } & \multicolumn{3}{|c|}{$\begin{array}{l}\text { Output amplified signal power in dual forward pumping with wavelength } 980 \mathrm{~nm}(\mathrm{~W} / \mathrm{nm}) \\
\text { with the constant input signal power }=0.001 \mathrm{mw}\end{array}$} & \multirow[t]{2}{*}{ Remarks } \\
\hline & EDFA length $10 \mathrm{~m}$ & EDFA length $50 \mathrm{~m}$ & EDFA length $120 \mathrm{~m}$ & \\
\hline Pump power $=0.22 \mathrm{w}$ & 0.009 & 0.005 & 0.0025 & S/g power decreases \\
\hline Pump power $=0.62 \mathrm{w}$ & 0.0023 & 0.0145 & 0.01 & S/g power decreases \\
\hline Pump power $=1 \mathrm{w}$ & 0.03 & 0.024 & 0.0165 & S/g power decreases \\
\hline Remarks & S/g power increases & S/g power increases & S/g power increases & \\
\hline
\end{tabular}

TABLE 3: Results: output amplified signal power of EDFA with Tri-forward pumping using wavelength $980 \mathrm{~nm}$ for different pumping power and different EDFA length.

\begin{tabular}{|c|c|c|c|c|}
\hline \multirow[t]{2}{*}{ Pump power } & \multicolumn{3}{|c|}{$\begin{array}{l}\text { Output amplified signal power in EDFA with Tri-forward pumping using wavelength } 980 \mathrm{~nm}(\mathrm{~W} / \mathrm{nm}) \\
\text { with the constant input signal power }=0.001 \mathrm{mw}\end{array}$} & \multirow[t]{2}{*}{ Remarks } \\
\hline & EDFA length $10 \mathrm{~m}$ & EDFA length $50 \mathrm{~m}$ & EDFA length $120 \mathrm{~m}$ & \\
\hline $\begin{array}{l}\text { Pump power }= \\
0.22 \mathrm{w}\end{array}$ & 0.006 & 0.0059 & 0.00256 & $\begin{array}{l}\text { S/g power } \\
\text { decreases }\end{array}$ \\
\hline $\begin{array}{l}\text { Pump power }= \\
0.62 \mathrm{w}\end{array}$ & 0.018 & 0.014 & 0.0098 & $\begin{array}{l}\text { S/g power } \\
\text { decreases }\end{array}$ \\
\hline $\begin{array}{l}\text { Pump power }= \\
1 \mathrm{w}\end{array}$ & 0.0286 & 0.021 & 0.017 & $\begin{array}{l}\text { S/g power } \\
\text { decreases }\end{array}$ \\
\hline Remarks & S/g power increases & S/g power increases & S/g power increases & \\
\hline
\end{tabular}

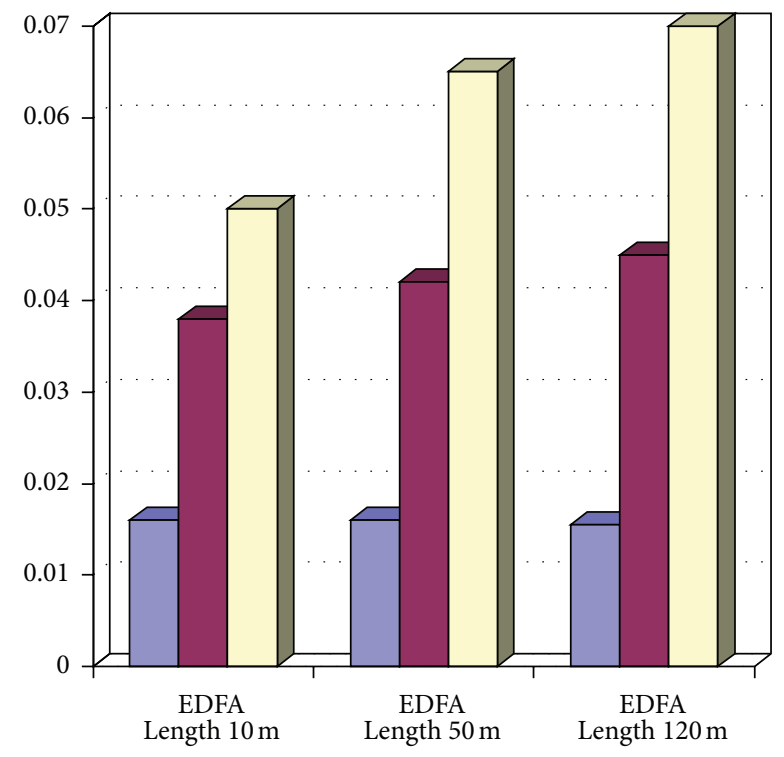

FIGURE 7: Bar chart output amplified signal power in dual pumping with wavelength $980 \mathrm{~nm}(\mathrm{~W})$ with the constant input signal power $=0.001 \mathrm{mw}$.

to population inversion, and population inversion is due to pump power.

Figures 7, 8, and 9 show the bar chart analysis of amplified signal output power for dual forward and backward pumping, dual forward pumping, and Tri-forward pumping techniques.

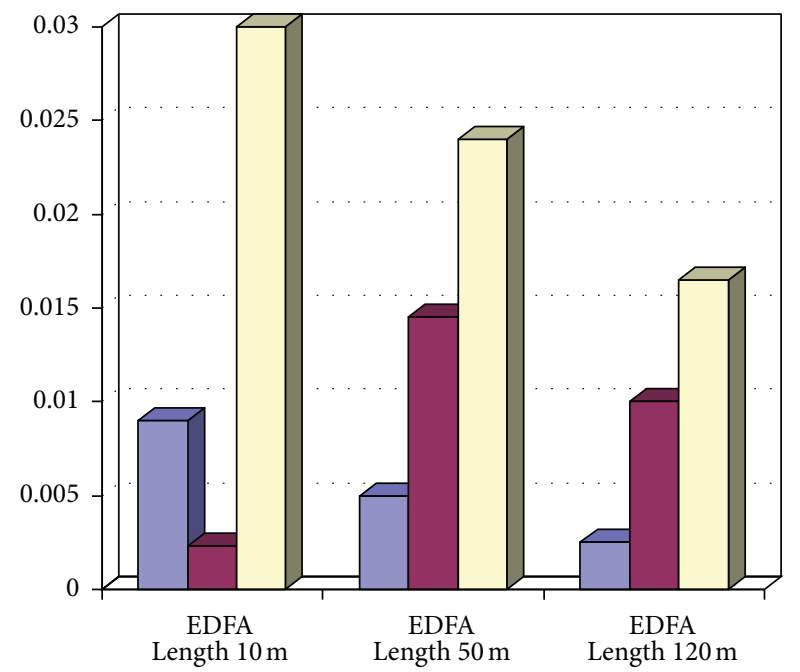

FIGURE 8: Output amplified signal power in dual forward pumping with wavelength $980 \mathrm{~nm}(\mathrm{~W})$ with the constant input signal power $=0.001 \mathrm{mw}$.

\section{Conclusion and Future Aspects}

To summarize, we have simulated the EDFA model with dual pumping and dual forward pumping and Tri-forward pumping scheme using $980 \mathrm{~nm}$ wavelength. The results gain and output ASE noise were compared and analyzed. Advancements in EDFA performance have allowed for longer fiber links between regenerators. To reduce the cost of regeneration efforts are ongoing to improve amplifier performance. This 


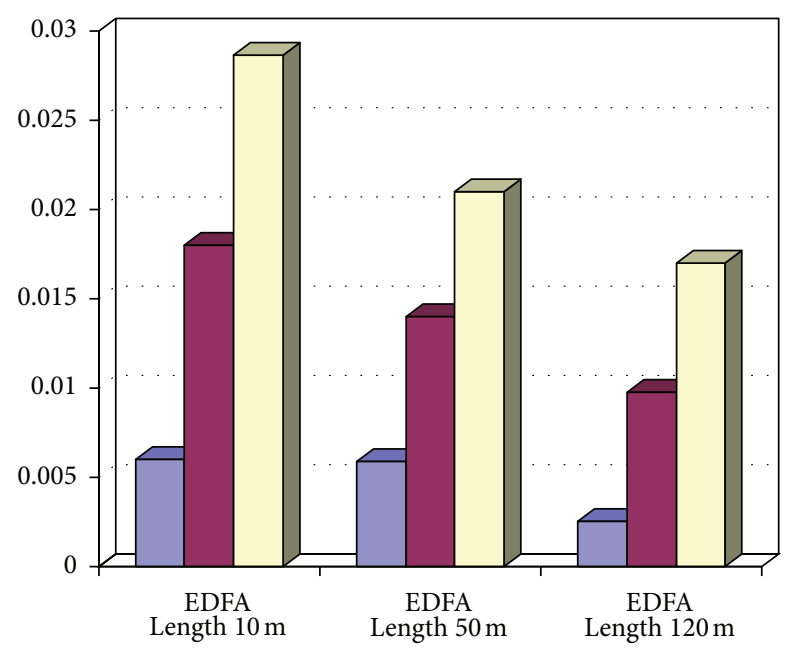

Figure 9: Output amplified signal power in Tri-forward pumping with wavelength $980 \mathrm{~nm}(\mathrm{~W})$ with the constant input signal power $=0.001 \mathrm{mw}$.

paper aims at modeling an EDFA with different pumping techniques to mitigate optical signal-to-noise (OSNR) and nonlinear effects in DWDM networks. Thus, we have shown that the proposed model of an EDFA utilizing various pumping techniques was successfully simulated using WDM. The analyzed model is applicable in network reconfiguration and multivendor networks and also addition of new services and wavelengths. The results have been compared with the conventional EDFA parameter values.

In future work, the model can be modified and enhanced further by gain flattening filters (GFF) based on advanced fiber Bragg gratings (FBG) which allow amplifier manufacturers to improve gain flatness. Advanced FBGs can be used to replace other GFF technologies in current-generation amplifier designs as a simple means to improve gain ripple. Similarly, new amplifier designs can take advantage of this technology to help and push the performance of nextgeneration amplifiers to new heights.

\section{References}

[1] M. N. Zervas, R. I. Laming, and D. N. Payne, "Efficient erbiumdoped fiber amplifiers incorporating an optical isolator," IEEE Journal of Quantum Electronics, vol. 31, no. 3, pp. 472-480, 1995.

[2] J. Hecht, Understanding Fiber Optics, Prentice Hall, Upper Saddle River, NJ, USA, 2002.

[3] P. C. Becker, N. A. Olsson, and J. R. Simpson, Erbium-Doped Fiber Amplifiers Fundamentals and Technology, Academic Press, San Diego, Calif, USA, 1999.

[4] A. Goel and R. S. Mishra, "Design of broadband EDFA next generation optical network," International Journal of Neural Networks and Applications, pp. 9-13, 2010.

[5] M. Horiguchi, "Erbium Doped optical fiber amplifiers pumped in $660 \mathrm{~nm}$ and $820 \mathrm{~nm}$ bands," IEEE Journal of Light Wave Technology, vol. 12, no. 5, 1994.

[6] A. Temmar, H. Ould Saadi, and A. Boutaleb, "Simulation based analysis of Erbium Doped Fiber Amplifier (EDFA)," Journal of Applied Sciences, vol. 6, no. 4, pp. 789-794, 2006.
[7] S. Novak and A. Moesle, "Analytic model for gain modulation in EDFAs," Journal of Lightwave Technology, vol. 20, no. 6, pp. 975-985, 2002.

[8] T. G. Hodgkinson, "Average power analysis technique for erbium-doped fiber amplifiers," IEEE Photonics Technology Letters, vol. 3, no. 12, pp. 1082-1084, 1991.

[9] P. Schiopu and F. Vasile, "The EDFA performance with gain versus pump power," in Proceedings of the 27th IEEE International Semiconductor Conference (CAS '04), pp. 241-244, October 2004.

[10] I. E. Araci and G. Kahraman, "Performance failure analysis of EDFA cascades in optical DWDM packet-switched networks," Journal of Lightwave Technology, vol. 21, no. 5, pp. 1156-1163, 2003.

[11] J. H. Jang, J. H. Jung, W. J. Lee, W. W. Yun, and K. K. Lee, "Implementation of automatic gain controlled bidirectional EDFA in WDM networks," in Proceedings of the IEEE Pacific Rim Conference on Lasers and Electro-Optcis, pp. 650-651, September 1999. 

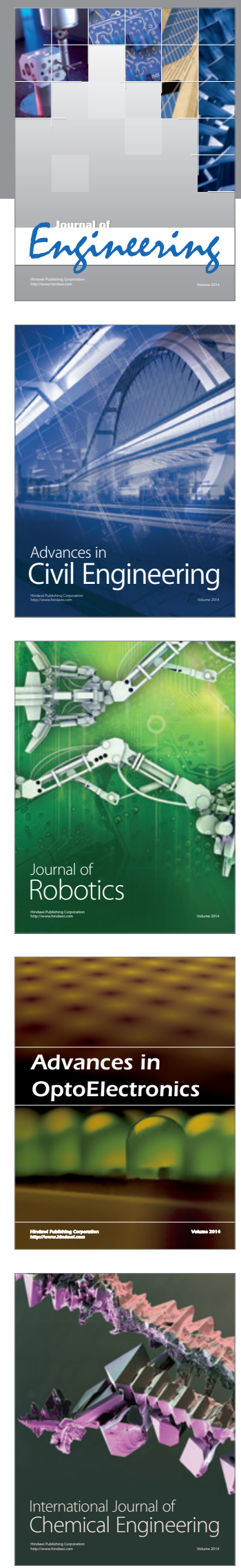

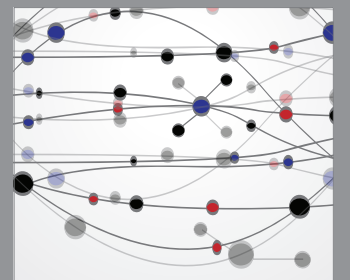

The Scientific World Journal
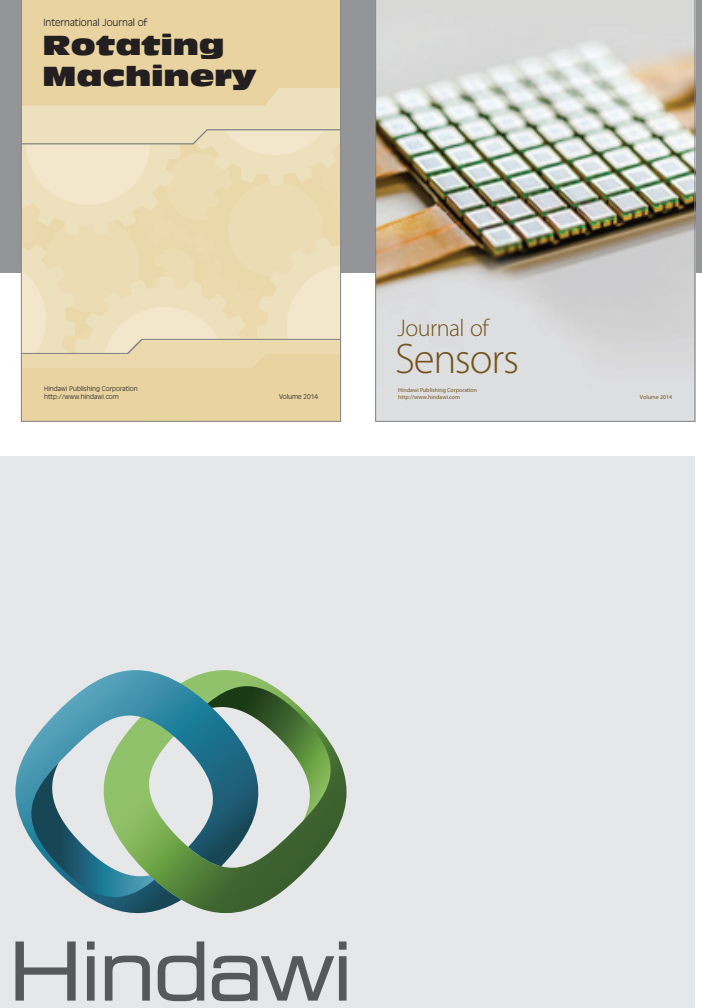

Submit your manuscripts at http://www.hindawi.com
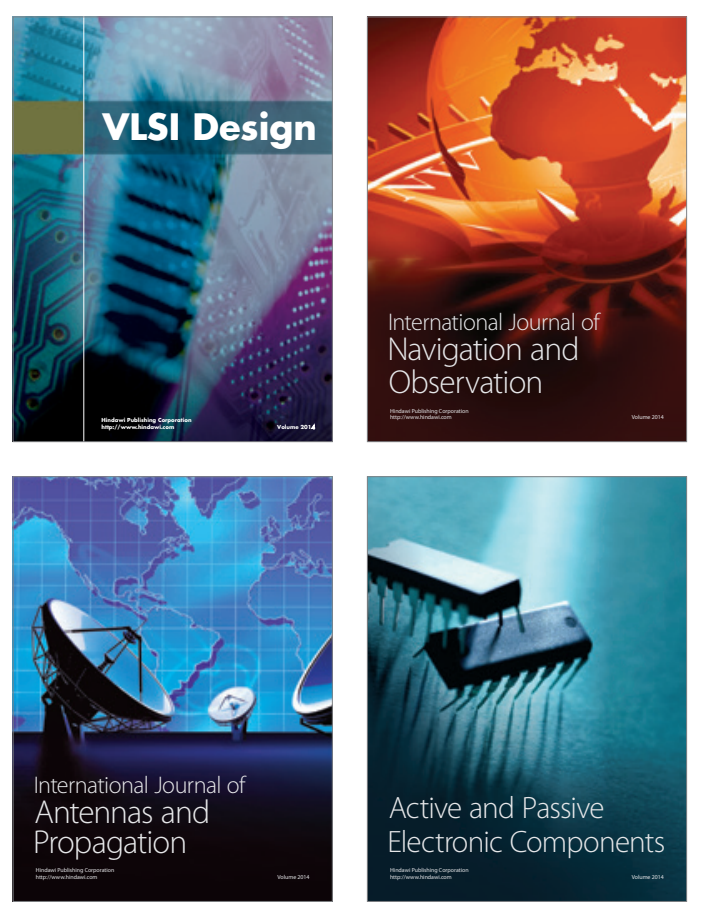
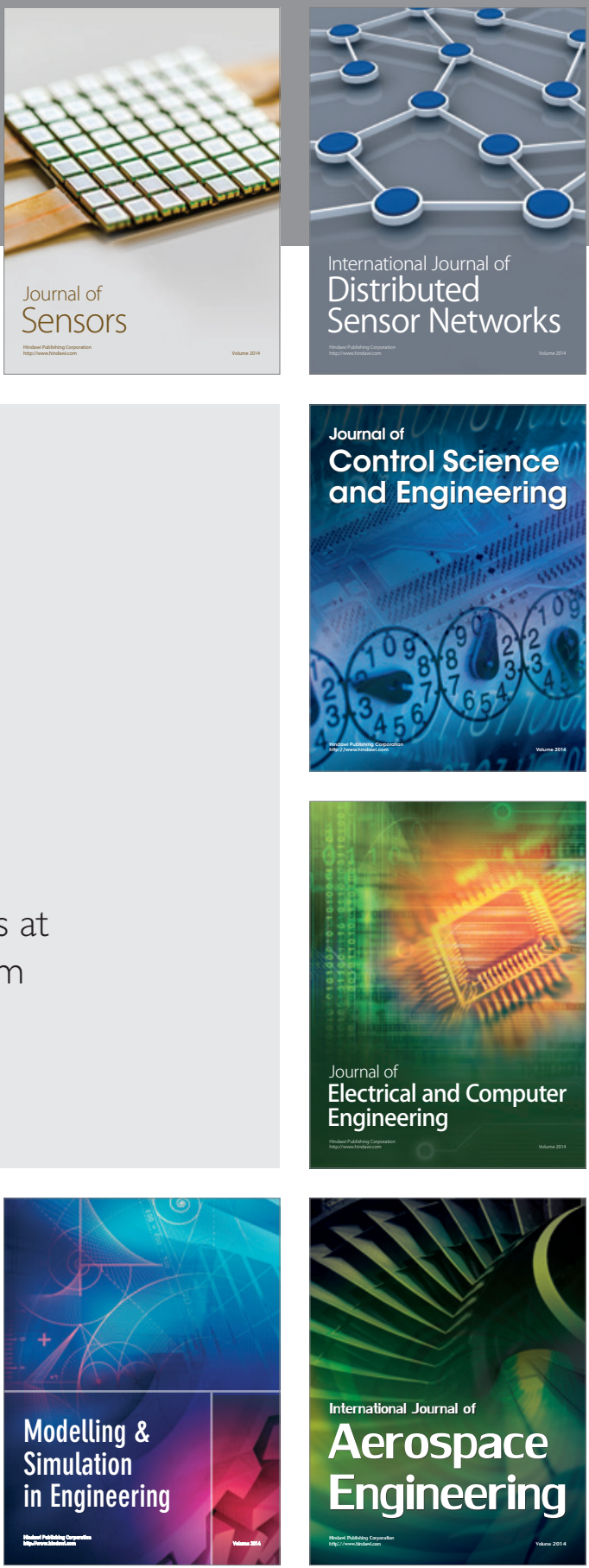

Journal of

Control Science

and Engineering
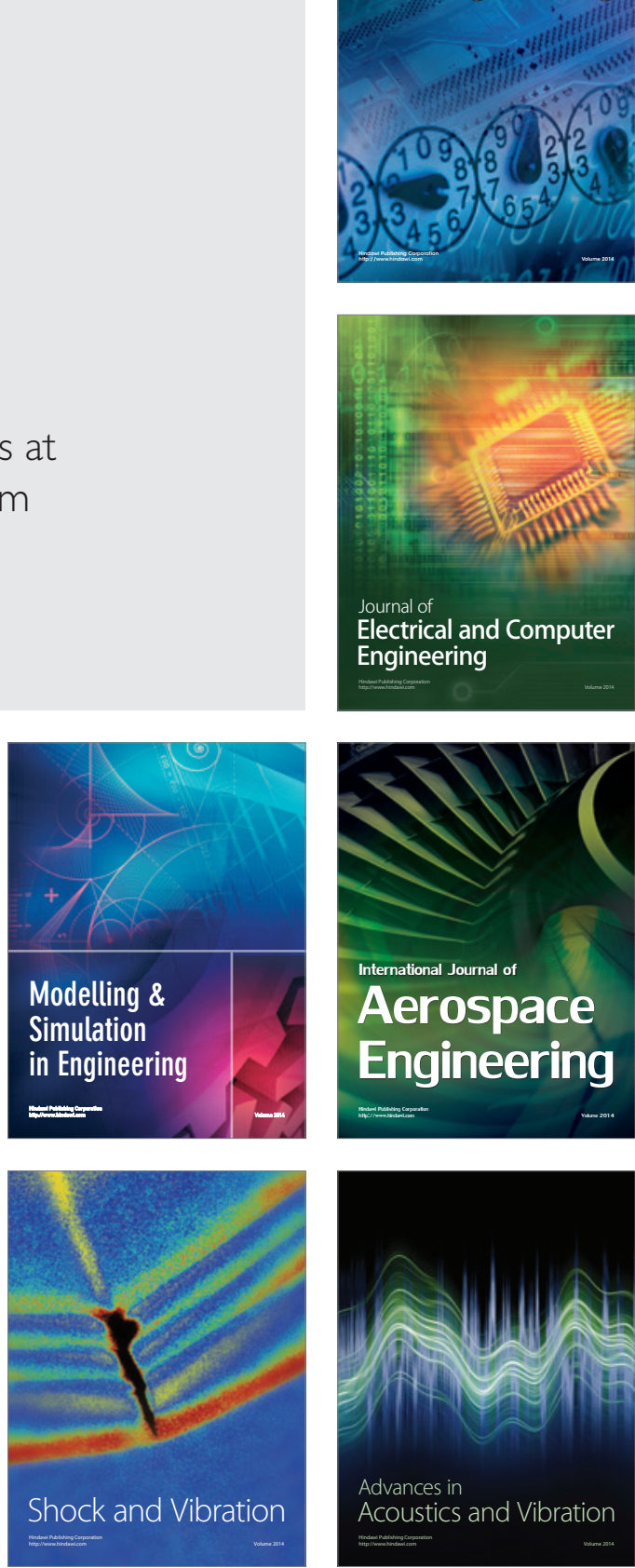